\title{
AKT Activation and Telomerase Reverse Transcriptase Expression are Concurrently Associated with Prognosis of Gastric Cancer
}

\author{
Takamitsu Sasaki $^{\mathrm{a}}$ Hiroki Kuniyasu $^{\mathrm{b}}$ Yi Luo $^{\mathrm{b}}$ Misaho Kitayoshi $^{\mathrm{b}}$ Eriko Tanabe ${ }^{\mathrm{b}}$ \\ Daisuke Kato $^{a}$ Satoshi Shinya ${ }^{a}$ Kiyomu Fujii ${ }^{b}$ Hitoshi Ohmori $^{b}$ Yuichi Yamashita $^{a}$ \\ ${ }^{a}$ Department of Gastroenterolgical Surgery, Fukuoka University School of Medicine, Fukuoka, b Department of Molecular \\ Pathology, Nara Medical University, Kashihara, Japan
}

\section{Key Words}

AKT · TERT · Telomerase $\cdot$ Prognosis

\begin{abstract}
AKT is a protein in the phosphatidylinositol-3 kinase (PI3K) pathway and associated with diverse pro-tumoral responses. Activation of the human telomere reverse transcriptase (hTERT) is one of AKT's tumorigenic effects. In this study, the significance of AKT phosphorylation and hTERT on prognosis of gastric cancer were examined. AKT activation by epidermal growth factor increased hTERT expression and telomerase activity. In contrast, AKT inactivation by inhibitors and knockdown decreased hTERT expression and telomerase activity in MKN28 gastric cancer cells. In 40 gastric cancer tissues, significant correlations were found among the levels of phosphorylated AKT (pAKT), hTERT expression, and telomer length. The pAKT levels or the levels of PAKT/hTERT were not associated with clinicopathological parameters, including stage and nodal metastasis. However, survival rates of the pAKT-high patients or the PAKT-high and hTERT-high patients were significantly poorer than those in other patients. These findings suggest that AKT and hTERT are good molecular targets for the treatment of gastric cancer.
\end{abstract}

Copyright $\odot 2013$ S. Karger AG, Basel

\section{Introduction}

The telomere is a repetitive 'TTAGGG' sequence present at the ends of eukaryotic chromosomes to maintain and protect their integrity [1]. As cells divide, the telomere is shortened in length; thus, the length of the telomere behaves like a marker of the division limit for cells and/or for cell death [2]. In stem cells and cancer cells, the telomere is elongated by telomerase activity, which enables them to divide endlessly [3].

The catalytic subunit of human telomeras reverse transcriptase (hTERT) is responsible for telomerase activity

\begin{tabular}{ll}
\hline Abbreviations used in this article \\
\hline PI3K & $\begin{array}{l}\text { Phosphatidylinositol-3 kinase } \\
\text { hTERT }\end{array}$ \\
hAman telomerase reverse transcriptase \\
pAKT & $\begin{array}{l}\text { phosphorylated AKT } \\
\text { Epidermal growth factor }\end{array}$ \\
EGF & $\begin{array}{l}\text { phosphatase and tensin homolog deleted on } \\
\text { PTEN }\end{array}$ \\
mTOR & $\begin{array}{l}\text { mammalian target of rapamysin } \\
\text { mF }\end{array}$ \\
NF & nuclear factor \\
EMT & epithelial-mesenchymal transition \\
VEGF & vascular endothelial growth factor
\end{tabular}


and telomere elongation and is suppressed in differentiated cells [4]. In our previous study, we reported that telomere shortening is a significant factor for the induction of TERT expression in gastric mucosa [5].

$\mathrm{AKT}$ is a protein in the phosphatidylinositol-3 kinase (PI3K) pathway. Stimulation of receptor tyrosine kinases or G-proteins activates $\mathrm{PI} 3 \mathrm{~K}$, which in turn activates AKT. AKT phosphorylation is maintained by heat shock protein 90 , and AKT is dephosphorylated by protein phosphatase $2 \mathrm{~A}$. Thus, AKT is involved in signaling mediated by various growth factors and cytokines. In particular, insulin-like growth factor-1, epidermal growth factor receptor, and human epidermal growth factor receptor 2, which are important in cancer-progression, activate AKT $[6,7]$. Hence, AKT is a one of biomarkers for predicting metastasis of human gastrointestinal cancer [8].

The phosphorylation of AKT modulates signals from phosphatase, tensin homolog deleted on chromosome 10 (PTEN), and the mammalian target of rapamycin to provide diverse effects on cells [9]. In this regard, AKT1 is recognized as an apoptotic inhibitor, which enhances cancer promotion. Phosphorylation via AKT inactivates $\mathrm{Bcl}-2$ antagonist of cell death resulting in its dissociation from Bcl-2. Nuclear factor $\kappa \mathrm{B}$ is also activated by AKT, which in turn up-regulates transcription of many survival genes [10]. AKT also induces angiogenesis through the up-regulation of vascular endothelial growth factor (VEGF) [11]. The AKT-microRNA regulatory network suggests that microRNA-mediated gene regulation interacts with the AKT signal pathway [12]. Hence, the expression of AKT is a pivotal tumorigenic factor and AKT is recognized as a relevant molecular target of cancer treatment [7].

The activity of hTERT is regulated by hTERT expression and phosphorylation. Protein kinase $\mathrm{C}$ and AKT can phosphorylate hTERT $[13,14]$. AKT phosphorylation of hTERT induces intranuclear translocation of hTERT and, subsequently, activates hTERT. In contrast, ring finger protein 1, an E3 ubiquitin ligase, decreases the activity of hTERT by ubiquitylation [15].

In the present study, AKT phosphorylation is correlated with clinicopathological parameters such as TERT expression and telomerase activity in gastric cancer.

\section{Materials and Methods}

\section{Cell Culture and Reagents}

The human gastric cancer cell line MKN28 (kindly gifted from Professor Wataru Yasui, Hiroshima University, Japan) was main- tained in Dulbecco's modified essential medium (Sigma Chemical Co., St. Louis, Mo., USA) containing 10\% fetal bovine serum (Sigma Chemical Co.) at $5 \% \mathrm{CO}_{2}$ in air and $37^{\circ} \mathrm{C}$. Wortmannin and triciribine were from Biovision LTD. (Milpitas, Calif., USA), and human epidermal growth factor was from Peprotec EC LTD. (Rocky Hill, N.J., USA).

\section{Clinical Materials}

Forty gastric tissues (approximately $30 \mathrm{~mm}^{3}$ ) were randomly selected from cases diagnosed at the Department of Molecular Pathology, Nara Medical University, between 2001 and 2010. The tissues had been frozen quickly in liquid nitrogen and stored at $-80^{\circ} \mathrm{C}$. The tissue contents were confirmed by microscopic observation of the adjacent tissues, which were prepared for histopathological examination.

\section{Short Interference RNA}

FlexiTube short interference RNAs (siRNAs) for AKT was purchased from Qiagen Genomics, Inc. (Bothell, Wash., USA). All Stars Negative Control siRNA was used as control (Qiagen Genomics, Inc.). Cells were transfected with $50 \mathrm{nM}$ siRNA for each gene using Lipofectamine 2000 (Invitrogen Corp., Carlsbad, Calif., USA) according to the manufacturer's instructions.

\section{Quantitative Reverse Transcription-Polymerase Chain}

Reaction ( $q R T-P C R)$

Total RNA $(1 \mu \mathrm{g})$ was used for cDNA synthesis with the ReverTra Ace qPCR RT Kit (Toyobo, Osaka, Japan). qRT-PCR were performed on the StepOne Real-Time PCR System (Applied Biosystems, Foster City, Calif., USA) using the Fast SYBR Green Master Mix (Applied Biosystems) and analyzed by employing the relative standard curve quantification method. The PCR parameters were set according to the manufacturer's instructions and the beta-actin mRNA level was used as internal control. All amplifications were evaluated by melting curve analysis and PCR products were electrophoresed on $2 \%$ agarose gels. All PCRs were performed at least in triplicate. Primer sets were purchased from Santa-Cruz.

\section{Immunoblot Analysis}

Cell lysates were prepared as described previously [16]. Next, $25 \mu \mathrm{g}$ (total protein) from lysates were electrophoresed on a $12.5 \%$ sodium dodecyl sulfate-polyacrylamide gel followed by electrotransfer to nitrocellulose membranes, which were subjected to immunoblot analysis. The membranes were incubated with primary antibodies and then probed with the appropriate peroxidase-conjugated secondary IgG antibodies (Medical and Biological Laboratories, Nagoya, Japan). Anti-tubulin antibody (LifeSpan Biosciences, Inc., Seattle, Wash., USA), anti-AKT antibody (Rockland Immunochemicals Inc., Gilbertsville, Pa., USA) and anti-phosphorylated AKT antibody (pSer463, Biorbyt, Cambridge, UK) were used as primary antibodies. The immune complex was visualized with the Enhanced Chemiluminescence Western-blot detection system (Amersham, Aylesbury, UK).

\section{Enzyme-Linked Immunosorbent Assay (ELISA)}

The lysates prepared as described above were also used for ELISA. Concentrations of pAKT and TERT were evaluated using specific ELISA kits, i.e., Akt (pS473) ELISA kit (Abcam, Cambridge, 
Fig. 1. Relationship between pAKT, hTERT, and telomerase activity. (a) Levels of pAKT protein and hTERT mRNA and telomerase activity were compared in MKN28 cells treated with EGF $(10 \mathrm{ng} / \mathrm{ml})$, wortmannin $(10 \mathrm{nM})$, triciribine $(10 \mu \mathrm{M})$, and siRNA against hTERT. Tubulin was used as loading controls. (b-d) Levels of pAKT protein and TERT mRNA and telomerase activity were compared in 40 gastric cancer cases. Levels of telomere length, pAKT and hTERT were represented as a relative value to that in peripheral blood lymphocytes, which was set to 1.0 .

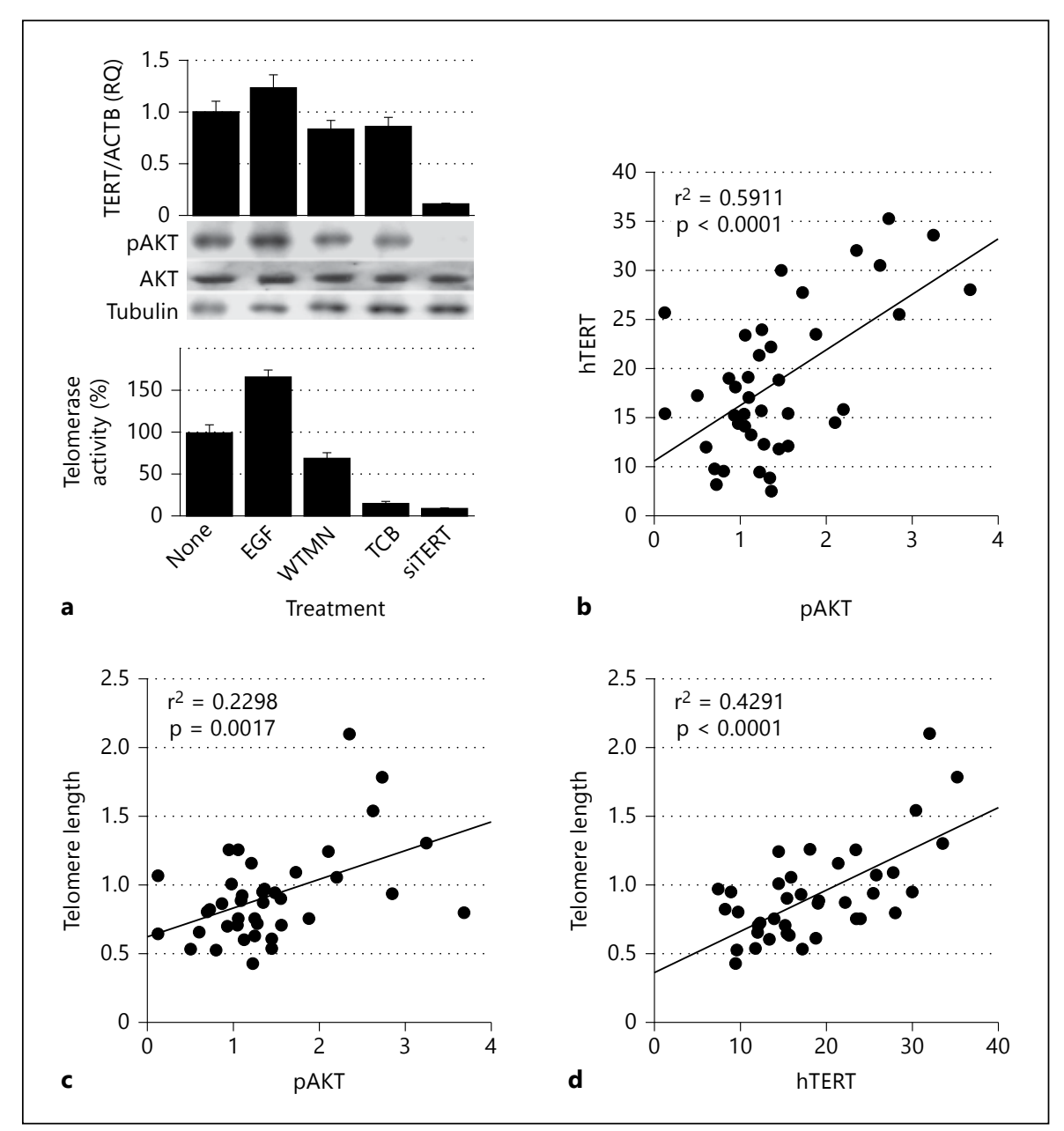

UK) and human telomerase reverse transcriptase (HTERT) ELISA kit (Oxford Expression Technology, Oxford, UK), according to the manufacturers' instructions.

Telomerase Activity and Telomere Length

Telomerase activity was examined by using the Quantitative Telomerase Detection Kit (US Biomax Inc., Rockville, Md., USA). The telomere length was examined by employing the TeloTAGGG Telomere Length Assay (Roche Applied Science, Indianapolis, Ind., USA). These kits were used according to the manufacturers' instructions.

\section{Statistical Analysis}

Statistical analyses of experimental data were carried out using the Spearman $r$ test and ANOVA. The positivities of pAKT and TERT were compared using the two-tailed chi-squared test (InStat, Graphpad Software Inc., La Jolla, Calif., USA). Survival analysis was performed by using the Kaplan-Meier method along with the Logrank test. Univariate and multivariate analyses were calculated by using Cox's hazard model (SPSS Statistics, IBM Japan, Tokyo, Japan). Statistical significance was defined as a two-sided p value of less than 0.05 .

\section{Results}

To examine the effect of AKT phosphorylation on hTERT expression and telomerase activity, MKN28 gastric cancer cells were analyzed after treatment under different conditions (fig. 1a). Epidermal growth factor stimulated AKT phosphorylation and increased hTERT expression and telomerase activity. In contrast, wortmannin, a PI3K inhibitor, triciribine, an AKT inhibitor, and siRNA-induced AKT knockdown inhibited AKT phosphorylation and decreased hTERT expression and telomerase activity. Total AKT protein levels were not affected by any treatment. These results suggest that AKT phosphorylation is associated closely with hTERT expression and telomerase activity.

Phospho-AKT (pAKT) levels, hTERT protein levels, and telomerase activity were examined in 40 cases of gastric cancer (fig. 1b-d). The pAKT level correlated with the hTERT level and telomerase activity, and the hTERT level correlated with telomerase activity $(\mathrm{p}<0.0001, \mathrm{p}=$ 
Table 1. Correlation of pAKT levels with clinicopathological parameters

\begin{tabular}{lccc}
\hline Parameter & \multicolumn{2}{l}{ pAKT } & \multirow{2}{*}{ p value } \\
\cline { 2 - 3 } & low & high & \\
\hline Differentiation & & & \\
tub1 & 5 & 6 & \\
tub2 & 8 & 5 & \\
por1 & 0 & 2 & \\
por2 & 5 & 5 & NS \\
sig & 2 & 2 & \\
Primary tumor & 4 & & \\
pT1 & 3 & 3 & NS \\
pT2 & 6 & 7 & \\
pT3 & 7 & 9 & NS \\
pT4 & & & \\
Nodal metastasis & 7 & 6 & \\
pN0 & 13 & 14 & \\
pN1-2 & & & \\
Pathological stage & 5 & 3 & NS \\
I & 4 & 4 & \\
II & 6 & 5 & \\
IIIA & 5 & 3 & \\
IIIB & 0 & 5 & \\
IV & 13 & 9 & \\
Status & 71 & \\
Survive & & & \\
Dead & & & \\
\hline
\end{tabular}

The pathological parameters were evaluated according to Japanese Classification of Gastric Cancer.

0.0017 , and $\mathrm{p}<0.0001$, respectively). These correlations were compatible to those found in figure la.

Next, pAKT levels were compared with clinicopathological parameters (table 1). Parameters in the pAKTHigh cases showed no significant difference when compared with those in the pAKT-Low cases. However, survival analysis showed that the pAKT-High cases had a significantly poorer prognosis than pAKT-Low cases (fig. 2a, $\mathrm{p}=0.0498$ ).

Next, the cases were divided into 3 categories, i.e., pAKT high and TERT high (Both High), pAKT low and TERT low (Both Low), and other cases (Intermediate), to examine the concurrent effect of pAKT and hTERT on disease progression (table 2). As shown in figure $2 c$, the both high cases were the 9 highest cases of the product of pAKT by hTERT. In contrast, the both low cases were the 7 lowest cases of the product. These cases were distinguishable by distribution of the products. Parameters of the Both-High cases showed no significant differences compared to those of the Both-Low cases. However, the
Table 2. Correlation of pAKT and hTERT levels with clinicopathological parameters

\begin{tabular}{lllll}
\hline Parameter & \multicolumn{2}{l}{ pAKT and hTERT } & \multirow{2}{*}{ p value } \\
\cline { 2 - 3 } & both high & intermed & both low & \\
\hline Differentiation & & & & \\
$\quad$ tub1 & 2 & 8 & 1 & \\
tub2 & 2 & 7 & 4 & \\
por1 & 0 & 2 & 0 & \\
por2 & 4 & 4 & 2 & NS \\
sig & 1 & 3 & 0 & \\
Primary tumor & & & & \\
pT1 & 1 & 1 & 3 & \\
pT2 & 1 & 5 & 0 & \\
pT3 & 3 & 8 & 2 & \\
pT4 & 4 & 10 & 2 & NS \\
Nodal metastasis & & & & \\
pN0 & 2 & 6 & 5 & \\
pN1-2 & 7 & 18 & 2 & \\
Pathological stage & & & & \\
I & 1 & 4 & 3 & \\
II & 2 & 5 & 1 & \\
IIIA & 2 & 7 & 2 & \\
IIIB & 1 & 6 & 1 & \\
IV & 3 & 2 & 0 & \\
Status & 3 & 14 & 5 & \\
Survive & 6 & 10 & 2 & \\
Dead & & & \\
\hline
\end{tabular}

The pathological parameters were evaluated according to Japanese Classification of Gastric Cancer.

survival analysis showed that the Both-High cases had a significantly poorer prognosis than the Both-Low and Intermediate cases (fig. 2b, $\mathrm{p}=0.0339$ ), and the Both-Low cases had a significantly better prognosis than the Intermediate cases. Thus, the levels of pAKT and TERT could be useful prognostic markers.

Finally, the significance of pAKT levels or pAKT/ hTERT levels was examined by univariate and multivariate analyses to compare stage and nodal metastasis (Table 3). Nodal metastasis emerged as an independent factor for the prognosis, whereas pAKT levels, pAKT/hTERT levels, and stage were dependent factors.

\section{Discussion}

In our study, the pAKT level or pAKT/hTERT levels showed no association with any clinicopathological parameters; however, the levels correlated well with disease prognosis. 
Fig. 2. Survival analyses of 40 gastric cancer cases. (a) Overall survival of in 20 cases with higher pAKT levels (pAKT High) was compared with that of 20 cases with lower pAKT levels (pAKT Low) by using the Kaplan-Meier method. (b) Overall survivals were compared between 9 cases with high AKT and high TERT (Both High), 7 cases with low pAKT and low TERT (Both High), and 24 cases with intermediate expressions (Intermed) by using the Kaplan-Meier method. (c) Distribution of the products of pAKT value by hTERT value in both high group (left panel) and both low group (right panel).

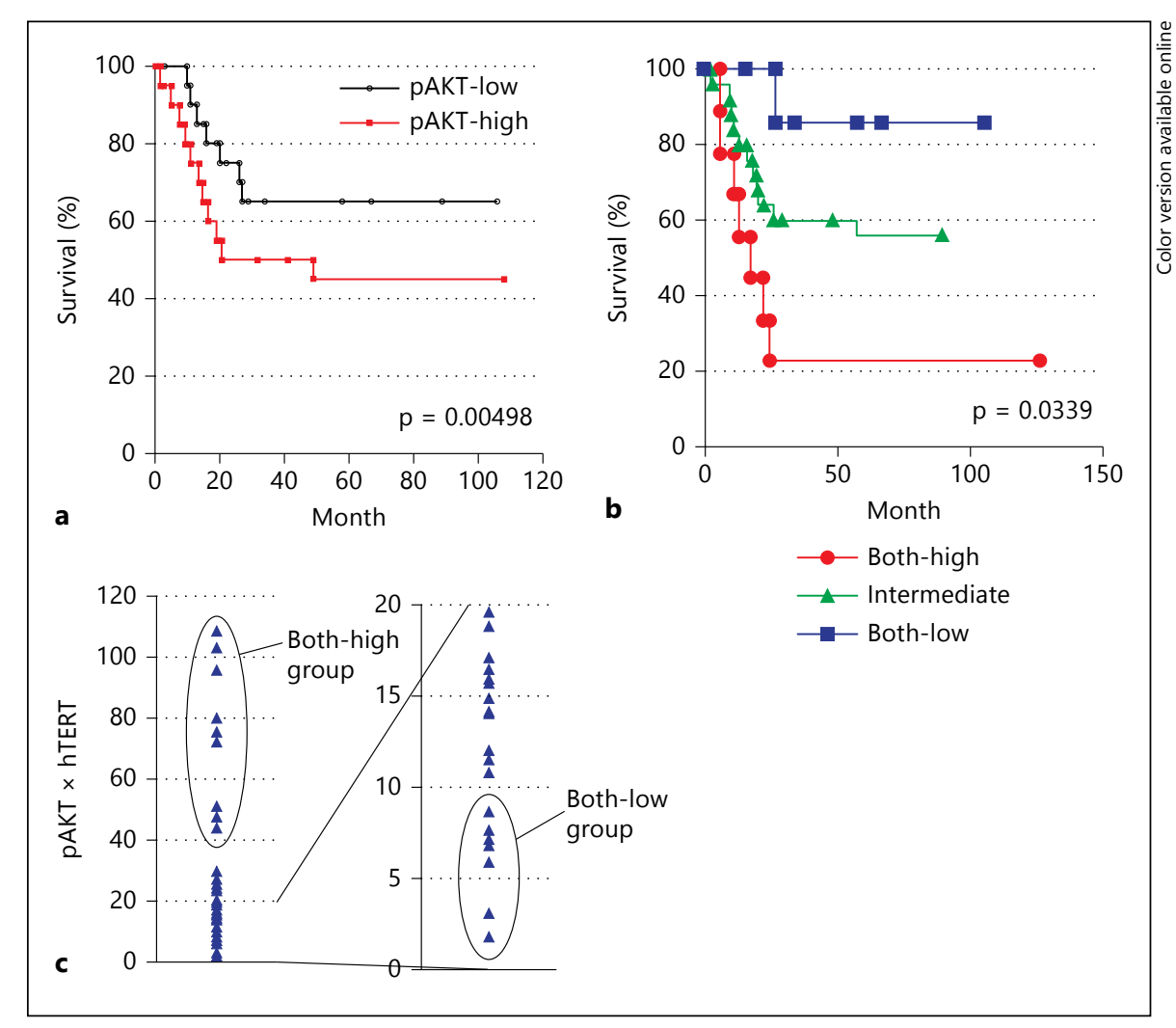

Table 3. Univariate and multivariate analyses of $\mathrm{pAKT}$ and/or hTERT levels

\begin{tabular}{llll}
\hline & Coefficient & $95 \%$ CI & p value \\
\hline Univariate analysis & & & \\
$\quad$ Stage & 0.0252 & $0.005-0.524$ & 0.0296 \\
Nodal metastasis & 7.613 & $1.727-33.562$ & 0.0008 \\
pAKT (High, Low) & 2.453 & $0.945-6.368$ & 0.0498 \\
pAKT/hTERT & 0.159 & $0.020-1.243$ & 0.0198 \\
Multivariate analysis & & & \\
Stage & 1.077 & $0.236-4.916$ & 0.9351 \\
Nodal metastasis & 0.132 & $0.028-0.627$ & 0.0108 \\
pAKT (high, low) & 0.572 & $0.236-1.389$ & 0.2172 \\
pAKT/hTERT & 0.793 & $0.267-2.353$ & 0.7995 \\
\hline
\end{tabular}

$\mathrm{CI}=$ Confidence interval. Both high, both low, intermed.

AKT is associated with cancer cell survival through altering Bcl-2 antagonist of cell death, p53, forkhead, nuclear factor $\kappa \mathrm{B}$, mammalian target of rapamycin, and PTEN [10] [17]. Moreover, dysregulated PTEN/PI3K/ AKT signaling interacts with the Wingless-INT pathway to induce epithelial-mesenchymal transition (EMT), which is usually associated with cancer stem cell-pheno- type and poor prognosis [18]. It has been recently reported that hTERT promotes transforming growth factor- $\beta$ and $\beta$-catenin-induced EMT by inducing $\beta$-catenin nuclear translocation and its transcriptional activity for vimentin expression [19]. Therefore, PTEN/PI3K/AKT signaling enhances EMT and stem cell phenotypes. In the present study, the association of AKT phosphorylation, TERT expression, and telomerase activity was confirmed in MKN28 gastric cancer cells and tissues of 40 gastric cancer patients. These associations could result in poor prognoses in cases with high pAKT levels or high pAKT/ hTERT levels. Multivariate analysis revealed that pAKT levels or $\mathrm{pAKT} / \mathrm{hTERT}$ levels were dependent prognostic factors. The examination of more gastric cancer cases is required to confirm the hypothesis that the EMT/stem cell phenotype affects disease progression.

Angiogenesis is an essential phenotype for cancer progression [20]. VEGF expression is associated closely with neovascularization and cancer progression in many malignancies. The PI3K/AKT pathway is one of the inducers of a VEGF response, which includes other inducers such as mitogen-activated protein kinase (extracellular signalregulated kinases or p38), Src, focal adhesion kinase, Rho family GTPases, and endothelial nitric oxide [21]. The 
PI3K/AKT pathway increases the secretion of VEGF from cancer cells by hypoxia-inducible factor 1-dependent and -independent mechanisms [22]. Therefore, AKT suppression could result in an anti-angiogenic effect on gastric cancer.
Our data showed that AKT and hTERT were widely expressed in gastric cancer. The concurrent expression of these 2 proteins at high levels is associated with a poor prognosis. These results suggest that AKT and hTERT are good molecular targets for the treatment of gastric cancer.

\section{References}

1 Blackburn EH: Switching and signaling at the telomere. Cell 2001;106:661-673.

-2 Lingner J, Cooper JP, Cech TR: Telomerase and DNA end replication: No longer a lagging strand problem? Science 1995;269:15331534.

-3 Stewart SA, Weinberg RA: Telomeres: Cancer to human aging. Annu Rev Cell Dev Biol 2006;22:531-557.

4 Autexier C, Lue NF: The structure and function of telomerase reverse transcriptase. Annu Rev Biochem 2006;75:493-517.

$\checkmark 5$ Kuniyasu H, Kitadai Y, Mieno H, Yasui W: Helicobactor pylori infection is closely associated with telomere reduction in gastric mucosa. Oncology 2003;65:275-282.

-6 Sukawa Y, Yamamoto H, Nosho K, Kunimoto H, Suzuki H, Adachi Y, Nakazawa M, Nobuoka T, Kawayama M, Mikami M, Matsuno T, Hasegawa T, Hirata K, Imai K, Shinomura Y: Alterations in the human epidermal growth factor receptor 2-phosphatidylinositol 3-kinase-v-AKT pathway in gastric cancer. World J Gastroenterol 2012;18:65776586.

7 Berg M, Soreide K: EGFR and downstream genetic alterations in KRAS/BRAF and PI3K/ AKT pathways in colorectal cancer: Implications for targeted therapy. Discov Med 2012; 14:207-214
8 Ng L, Poon RT, Pang R: Biomarkers for predicting future metastasis of human gastrointestinal tumors. Cell Mol Life Sci 2013;in press.

$\checkmark 9$ Cheung M, Testa JR: Diverse mechanisms of AKT pathway activation in human malignancy. Curr Cancer Drug Targets 2013;13:234-244.

10 Downward J: PI 3-kinase, AKT and cell survival. Semin Cell Dev Biol 2004;15:177-182.

11 Radisavljevic Z: AKT as locus of cancer angiogenic robustness and fragility. J Cell Physiol 2013;228:21-24.

$12 \mathrm{Xu} \mathrm{M}$, Mo YY: The AKT-associated microRNAs. Cell Mol Life Sci 2012;69:36013612.

13 Li H, Zhao L, Yang Z, Funder JW, Liu JP: Telomerase is controlled by protein kinase $\mathrm{Ca}$ in human breast cancer cells. J Biol Chem 1998;273:33436-33442.

14 Kang SS, Kwon T, Kwon DY, Do SI: Akt protein kinase enhances human telomerase activity through phosphorylation of telomerase reverse transcriptase subunit. J Biol Chem 1999; 274:13085-13090.

15 Kim JH, Park SM, Kang MR, Oh SY, Lee TH, Muller MT, Chung IK: Ubiquitin ligase MKRN1 modulates telomere length homeostasis through a proteolysis of hTERT. Genes Dev 2005;19:776-781.
16 Kuniyasu H, Oue N, Wakikawa A, Shigeishi $\mathrm{H}$ Matsutani N, Kuraoka K, Ito R, Yokozaki H, Yasui W: Expression of receptors for advanced glycation end-products (RAGE) is closely associated with the invasive and metastatic activity of gastric cancer. J Pathol 2002;196:163-170.

17 Populo H, Lopes JM, Soares P: The mTOR signalling pathway in human cancer. Int J Mol Sci 2012;13:1886-1918.

18 Karamitopoulou E: Tumor budding cells, cancer stem cells and epithelial-mesenchymal transition-type cells in pancreatic cancer. Front Oncol 2012;2:209.

19 Liu Z, Li Q, Li K, Chen L, Li W, Hou M, Liu T, Yang J, Lindvall C, Bjorkholm M, Jia J, Xu D: Telomerase reverse transcriptase promotes epithelial-mesenchymal transition and stem cell-like traits in cancer cells. Oncogene 2012; advance online publication.

20 Fidler IJ: The pathogenesis of cancer metastasis: The 'seed and soil' hypothesis revisited. 2003;3:453-458.

21 Claesson-Welsh L, Welsh M: VEGFA and tumour angiogenesis. J Intern Med 2013;273: 114-127.

22 Karar J, Maity A: PI3K/AKT/mTOR pathway in angiogenesis. Front Mol Neurosci 2011;4:51.

23 Japanese Gastric Cancer Association: Japanese classification of gastric carcinoma: 3rd English edition. Gastric Cancer 2011;14:101-112. 